\title{
Quantified characterization of phase structure in SBS modified asphalt based on microscopic morphological parameters
}

\author{
Aihong $\mathrm{KANG}^{\mathrm{a}}{ }^{,}$, Keke $\mathrm{LOU}^{\mathrm{a}}$, Changjiang $\mathrm{KOU}^{\mathrm{a}}$, Yingqian $\mathrm{WANG}^{\mathrm{a}}$, Zhengguang $\mathrm{WU}^{\mathrm{a}}$ \\ ${ }^{a}$ First affiliation, College of Civil Science and Engineering, Yangzhou University, Yangzhou, Jiangsu 225127, China \\ *Aihong KANG: kahyzu@163.com
}

\begin{abstract}
Polymer modified asphalt is a typical two-phase blends, and analyzing the phase structure is the most effective way to study its modification mechanism and modification effect. Four microscopic morphological parameters were extracted from fluorescence microscope microscopic images of SBS modified asphalt through professional image processing and analysis software. Then we analyzed each microscopic morphological parameter and corresponding phase structure. The results showed that the area-max is more suitable than the other parameters in characterizing the phase structure of SBS modified asphalt. Area-max $2500 \mu \mathrm{m}^{2}$ and $13000 \mu \mathrm{m}^{2}$ can be regarded as the critical point of phase transition. Modifier content is the most direct reason for the phase structure changes of polymer modified asphalt. The structure of the polymer phase would not change with the shearing time normally, but in the vicinity of the phase transition critical point, shearing time would have a significant influence on phase structure.
\end{abstract}

Keywords: SBS modified asphalt; microscopic parameters; phase structure; quantified characterization

\section{Introduction}

Due to it's outstanding performance, Polymer modified asphalt has been widely used in high-grade asphalt pavement ${ }^{(1-3)}$, the structure of polymer is an determinant factor of the modification effects ${ }^{(4)}$. Therefore, the study of structure of polymer modified asphalt has a great significance in practical applications ${ }^{(5-8)}$. By using the fluorescence microscopy, Weidong Huang and Lijun Sun ${ }^{(9)}$ in Tongji university find that polymer modified asphalt can be divided into three basic types based on the continuous of phase structure : The first one is a continuous asphalt phase and a discontinuous polymer phase; The second one is a continuous polymer phase and a discontinuous asphalt phase; The third one is continuous polymer phase and asphalt phase with interlock structure between them. Nahas ${ }^{(10)}$ thinks that besides the three kinds of structure above, the polymer which dissolves in asphalt will appear in the single phase structure as the polymer content is low. The matrix asphalt properties, the types of the polymer, contents and blending process have an effect on phase structure in some degree. As a result, a lot of research on phase structure of polymer modified asphalt mainly depend on visual inspection or a turning point of macroscopic properties to explain the change of phase structure ${ }^{(11)}$. They are all limited in qualitative description and it is difficult to be used in model and the evaluation of modified asphalt, the value is difficult to reflect in practical applications ${ }^{(12,13)}$. This paper used the microscopic images of SBS modified asphalt collected by the fluorescence microscope and the image analysis software to obtain microscopic parameters of modified asphalt, then the relationship between microscopic morphological parameters and phase structure will be analyzed and the appropriate microscopic parameters which can characterize the phase structure will be given too.

\section{Materials}

In this paper, Jinling 70\# matrix asphalt and the modifier (YH791, linear SBS polymers) which was produced in Yueyang petrochemical company were used to prepare a number of modified asphalt samples, the technical parameters are presented in table 2.1 and table 2.2. 
Tab 2.1 Basic technical parameters of matrix asphalt

\begin{tabular}{|c|c|}
\hline Performance indicators & Jinling 70 \# \\
\hline penetration (25 ${ }^{\circ} \mathrm{C} 100 \mathrm{~g}$, 5s $)$ & 74 \\
\hline Softening point & 46.6 \\
(ring and ball softening & \\
\hline point)/ ${ }^{\circ} \mathrm{C}$ & 257 \\
\hline Dynamic viscosity $(60 \quad 9 \mathrm{C} / \mathrm{Pa} \cdot \mathrm{s}$ & $>150$ \\
\hline ductility $(15 \quad 9 \mathrm{C} / \mathrm{cm}$ & \\
\hline
\end{tabular}

Tab 2.2 Technical indexes of SBS

\begin{tabular}{|c|c|}
\hline Project indicators & YH-791 \\
\hline structure & linear \\
\hline Block than (S/B) & $30 / 70$ \\
\hline Tear strength, kN/m & 35 \\
\hline Elongation at failure,\% & 700 \\
\hline
\end{tabular}

\section{Preparation of SBS polymer modified asphalt samples and pretreatment}

SBS polymer modified asphalt samples were prepared with Jinling 70 \# matrix asphalt and SBS polymers with different loading levels (3\%, 4\%, 5\%, 6\% and $7 \%$ by weight of modified asphalt) by mechanical mixing using a high shear mixer. under the condition of $170 \pm 3^{\circ} \mathrm{C}$, using the type of FA25 high-speed shear machine (Shanghai, China), samples can be obtained with A speed (10000 rpm) under the different shearing time, then preparing the samples through the test ${ }^{(14)}$ and waiting for collecting microscopic images after it was cooled down fully.

The acquisition of SBS modified asphalt microscopic image needs the fluorescence microscope. In order to enhance the visibility of the polymer phase and asphalt phase and extract microscopic parameters more convenient, we need to collect and gather the images after a series of process. In this paper, we make fluorescence microscopic images pretreatment by using the image acquisition software and MATLAB software which included: expanding the depth of field, image enhancement, object segmentation, filtering de-noising, binarization and so on.
Finally, parts of the images after processing are presented in figure 3.1 .

\section{The quantitative characterization of polymer morphology}

Microstructure of the polymer modified asphalt determines its macroscopic properties, Aihong Kang ${ }^{(15)}$ thinks that modifier particles such as shape, size, area percentage all affect the performance of the polymer modified asphalt. Daquan Sun ${ }^{(16)}$ find inflection point of the content of modifier - softening point curve by macroscopic test, and determine the phase transition point. Zuguang $\mathrm{Liu}^{(17)}$, through a fluorescence microscope pictures concluded that when the mass fraction of SBS is less than $5 \%$, the approximate globule were separated from each other, when the points reach $7 \%$ or more, the quality of the SBS form a continuous phase. Through the analysis of figure 2.1, we found that when the content is less than $4 \%$, SBS modified asphalt all assumes the circular point, when the content of $5 \%$ - $6 \%$, modifier grew into strips, when the content of $6 \%-7 \%$, partial mesh structure begin to form. These changes in morphology, will surely lead to the change of microscopic shape parameters, phase transformation point features should be similar to microscopic morphology characteristic parameter change point.

\section{1 selecting quantitative characterization of microscopic parameters}

In order to determine appropriate microscopic morphology parameters to characterize the change of the phase structure, this paper selected the aspect ( $\mathrm{L}_{\max }$ ), area percentage( $P$ ), fractal dimension (D), Area-max ( $\left.A_{\max }\right)$, this four microscopic morphology parameters were analyzed and as were presented in figure 4.1 to 4.4.

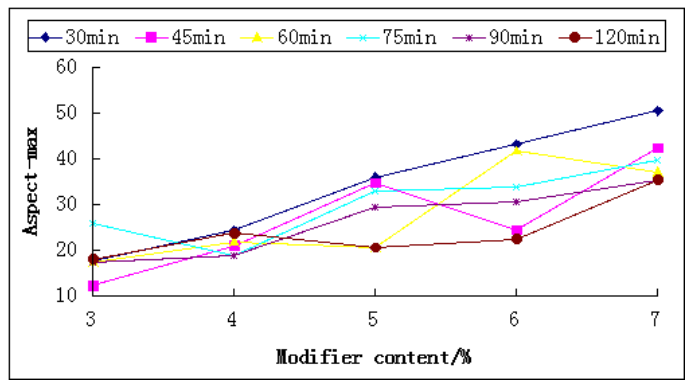

Fig4.1 Relations between $\mathrm{L}_{\max }$ and modifier content 


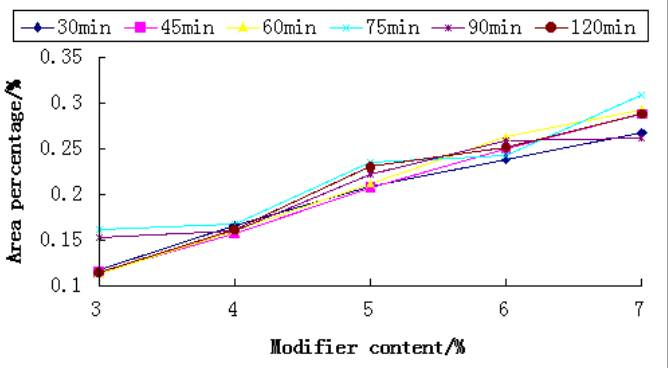

Fig4.2 Relations between P and modifier content

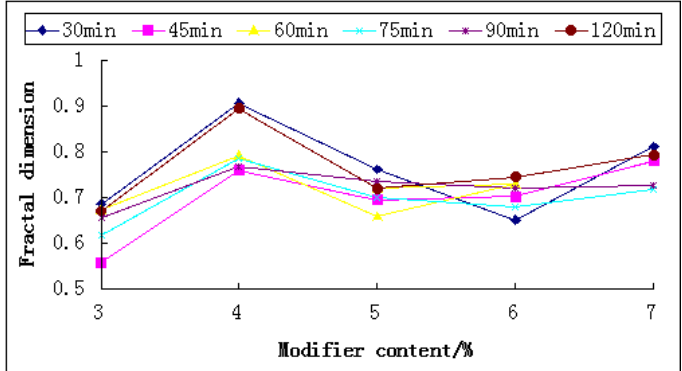

Fig4.3 Relations between D and modifier content

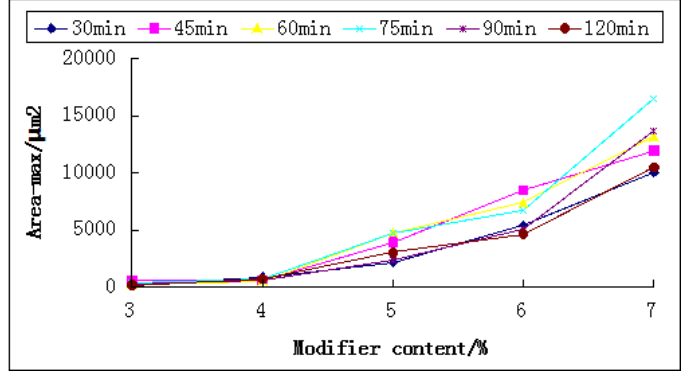

Fig4.4 Relations between $\mathrm{A}_{\max }$ and modifier content

We can see from the figures: Aspect increase gradually with the increase of content, but the variation is larger, it is difficult to determine the change point, there is little correlation between microstructure change; Modifier particles area percentage is roughly linear increase, when the content is less than $5 \%$, the growth of rate is slower than content is more than $5 \%$, growth rate decreases correlation with changes in the microstructure is small; Fractal dimension wave of change with the increase of content, do not tally with the microscopic structure change; When content is less than $4 \%$, Area-max slowly growth , when the content increase from $4 \%$ to $6 \%$, the change is accelerated, when the content increase from $6 \%$ to $7 \%$, it increases sharply, image clearly point and microstructure changes. Therefore, Area-max was selected as a characterization of morphology change of microscopic parameters.

\subsection{Based on analysis of microscopic parameters of morphology change}

According to the division of the microscopic parameters phase structure of polymer modified asphalt, a combination of collected microscopic images for finding out the morphology change point is required. The 2700 pictures according to the Area-max from small to large sort, and numbered in turn $N_{i}(i=1,2,3 \ldots \ldots, 2700)$. Then, all the microscopic images should be classified according to the morphology. According to the study, when $i<1390$, the image of asphalt and polymer dispersed structure is continuous. When $1390<i<2480$, a continuous two-phase or interlocking structure will form. When $i>2480$, a continuous polymer and asphalt phase dispersed structure will form, looking up the Area-max from the images in turn which were numbered $N_{1390}, N_{2480}$. The result is presented in figure 4.5 .

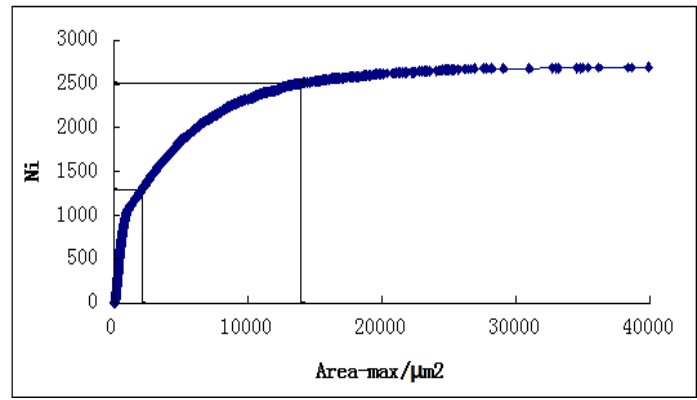

Fig4.5 Relations between area-max and picture number

To determine the critical point of phase transformation is polymer particles largest area of $2500 \mu \mathrm{m}^{2}$ and $13000 \mu \mathrm{m}^{2}$. When vision within the polymer particles biggest area of less than $2500 \mu \mathrm{m}^{2}$, asphalt is continuous but polymer is dispersed. When vision of the biggest polymer particles area is between $2500 \mu \mathrm{m}^{2}$ and $13000 \mu \mathrm{m}^{2}$, polymer modified asphalt gradually formed a two-phase continuous or interlock structure. When vision of the biggest polymer particles area is more than $13000 \mu \mathrm{m}^{2}$, polymer gradually forms a space network structure, and then phase gradually continued but asphalt is gradually dispersed. We can define that the first category is asphalt continuous and polymer dispersed, the second category is two-phase continues or interlock structure, the third category is polymer continuous and asphalt dispersed. That is: 


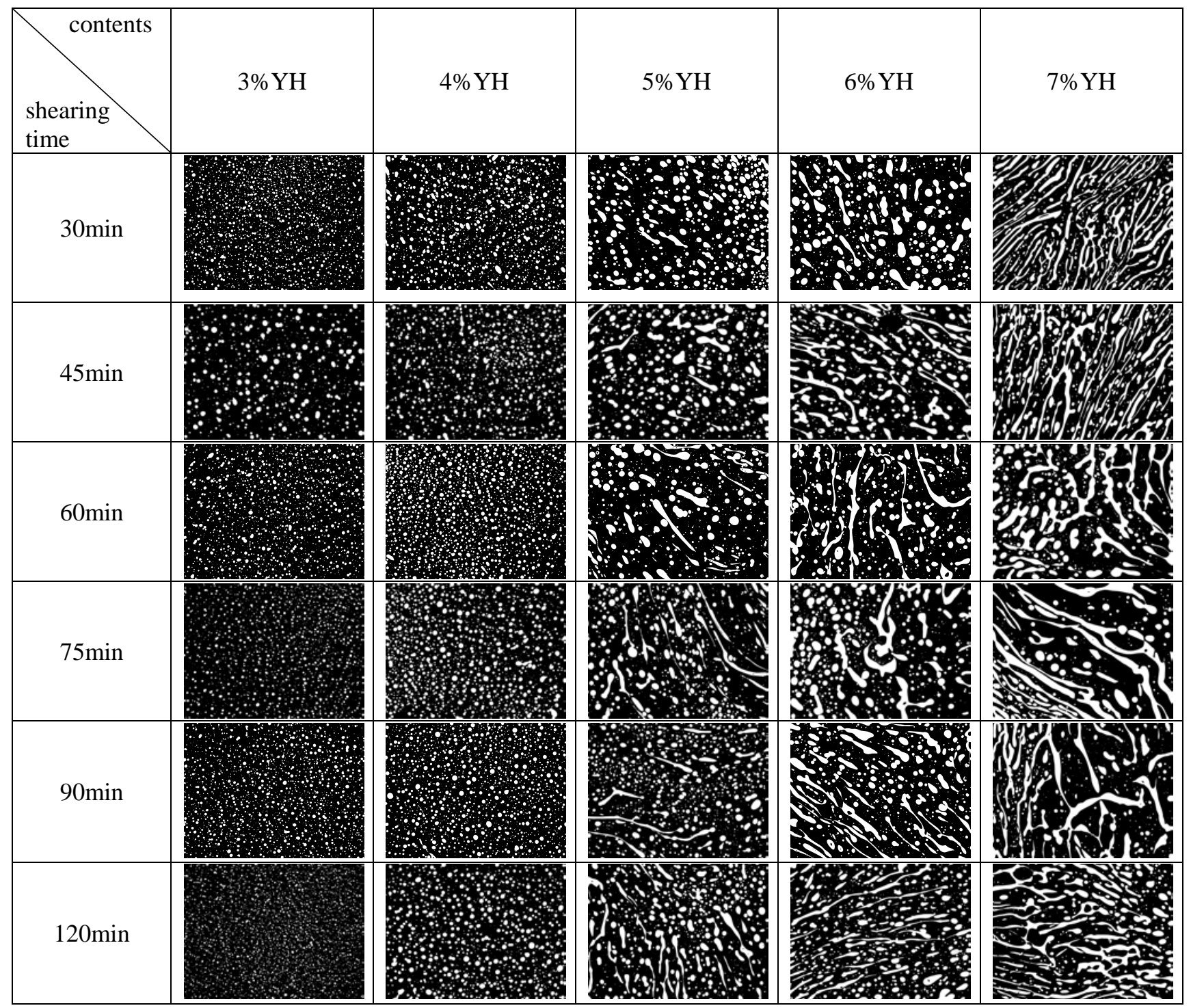

Fig3.1 Preprocessed images

the first category : $\quad \operatorname{Area}(\max ) \in(0,2500)$

the second category : Area $(\max ) \in(2500,13000)$

the third category : $\quad \operatorname{Area}(\max ) \in(13000, \infty)$

According to the test species used Jinling 70\# asphalt and the type of YH791 linear modifier, recommended value above is given. The tests the modifiers of DG, LG were conducted on respectly as well, the test results are shown in table 4.1.

The results showed that with different modifiers, using microscopic parameters expression transformation point has certain differences. But it can be found: even for different kinds of matrix asphalt and modifier, when the Area-max is less than $1500 \mu \mathrm{m}^{2}$, it can only form a asphalt continuous and polymer dispersed structure and it is difficult to form a continuous two-phase or interlocking structure. When the Area-max is more than $15000 \mu \mathrm{m}^{2}$, it can form a continuous
Tab 4.1 the critical value of phase structure between basis asphalt and different modifier

\begin{tabular}{|c|c|c|c|}
\hline $\begin{array}{c}\text { The } \\
\text { types of } \\
\text { modifier }\end{array}$ & $\begin{array}{c}\text { Continuous } \\
\text { asphalt but } \\
\text { dispersed } \\
\text { polymer }\end{array}$ & $\begin{array}{c}\text { Tow-phase } \\
\text { continues or } \\
\text { interlock } \\
\text { structure }\end{array}$ & $\begin{array}{c}\text { Continuous } \\
\text { polymer but } \\
\text { dispersed } \\
\text { asphalt }\end{array}$ \\
\hline YH & $(0,2500)$ & $\begin{array}{c}(2500, \\
13000)\end{array}$ & $\begin{array}{c}(13000, \\
\infty)\end{array}$ \\
\hline DG & $(0,2600)$ & $\begin{array}{c}(2600, \\
14000)\end{array}$ & $\begin{array}{c}(14000, \\
\infty)\end{array}$ \\
\hline LG & $(0,1500)$ & $(1500$, & $(15000$, \\
& & $15000)$ & $\infty)$ \\
\hline
\end{tabular}

polymer and asphalt dispersed structure.

4.3 Based on the macro performance analysis of phase structure change 
The macroscopic properties and microstructure of polymer modified asphalt are linked closely; the essence of the changes in the macroeconomic performance is the changes in microstructure. With the increase of the content of modifier, the macroscopic properties are directly affected, the microstructure will change affirmly. Isacsson ${ }^{(18)}$ believes that, with the increase of content of SBS modified asphalt, polymer modified asphalt phase structure is as follows: Continuous asphalt but dispersed polymer, tow-phase continues or interlock structure, continuous polymer but dispersed asphalt. The R.Blanco ${ }^{(19)}$ found that: There is a critical SBS content : CPi, when the content of SBS is greater than $\mathrm{CPi}$, it will be reversed. In order to broadly analyze the transformation point of polymer modified asphalt and the change regulation of phase state. In this study, rut factor in different content of polymer modified asphalt were tested respectively, which was presented in figure 4.6.

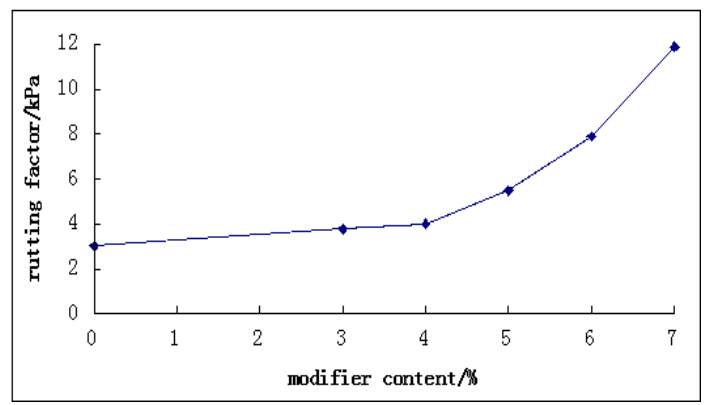

Fig4.6 Relations between rutting factor and modifier content

We can see from figure 4.6: when the modifier content is less than $4 \%$, the growth of rut factor is slightly and roughly accords to linear growth, and the polymer phase is dispersed. When the content in the range of $4 \%-6 \%$, rut factor of modified asphalt with the increasing of modifier content increase faster, it roughly present a exponential growth. Polymer phase and the asphalt phase are a continuous two-phase or interlocking structure. When the content of transition from $6 \%$ to $7 \%$, the trend of its rut factor shows a fastest growth, and polymer forms a continuous phase gradually. That is:

$$
\left\{\begin{array}{l}
\text { the first category: } \quad \text { Modifier } \in(0,4.5 \%) \\
\text { the second category : } \text { Modifier } \in(4.5 \%, 7 \%) \\
\text { the third category: } \quad \text { Modifier } \in(7 \%, \infty)
\end{array}\right.
$$

\subsection{Effects of shearing time on phase structure}

Disperse state of modifier in asphalt is affected by blending technology ${ }^{(20)}$, The difference of process parameters result in the same content of modifier produces different micro morphology. This paper analyzes one of the factors: Effects of shearing time on polymer phase structure, establishing the relationship curve about the Area-max and the shearing time, as were presented in figure 4.7-4.8.

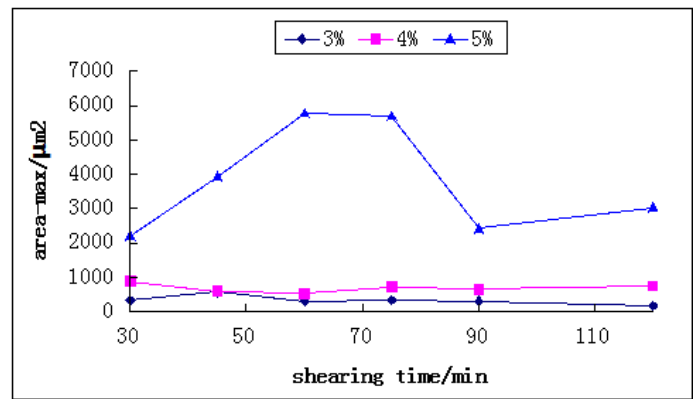

Fig4.7 Relations between area-max and shearing time

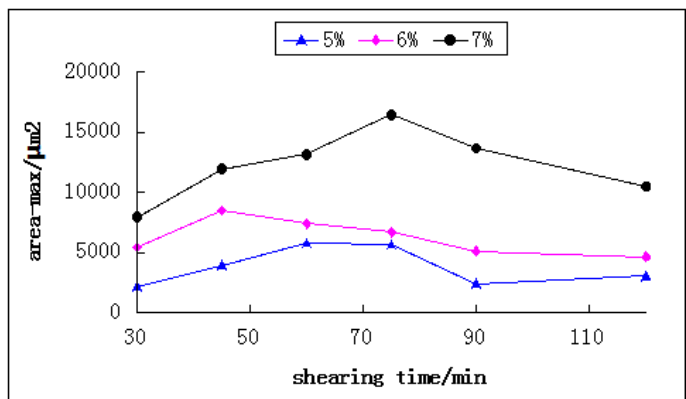

Fig4.8 Relations between area-max and shearing time

As we can see from figure 4.7-4.8, the Area-max will change with the growth of shearing time, and that is to say the shearing time influences the Area-max. When the content is less than $4 \%$, the Area-max will be less than $1000 \mu \mathrm{m}^{2}$ regardless of the length of shearing time, and the polymer phase can only form a dispersed phase that is a single phase and continuous system and shearing time will not cause the change of phase structure. When the content between $5 \%$ and $6 \%$, although the Area-max is changing, it does not cause changes of phase structure. When the content is $7 \%$, the phase structure changed with the growth of shearing time. Because of it, we can see that shearing time does not change the phase structure of polymer modified asphalt generally, but around the critical point of the phase transition, shearing time will have a certain impact on the phase structure.

\section{Conclusions}

After polymer and matrix asphalt have been blended, the composition and phase structure of asphalt became very complex. In the process of researching polymer modified asphalt phase structure, we need to consider the modified 
asphalt macroscopic properties and the subjective identify about microscopic image combined with the morphological parameters of fluorescence microscopy to indicate the phase structure quantitatively. The four kinds of microstructure parameters have been compared in this paper, we find that using Area-max to identify the phase structure of SBS modified asphalt is appropriate, and providing Area-max which are $2500 \mu \mathrm{m}^{2}$ and $13000 \mu \mathrm{m}^{2}$ to be the critical point of the phase transformation from the micro morphological parameters, and given the modifier content which is $4.5 \%$ and $7 \%$ as the critical point of the phase transformation at the macro level. The modifier content is the most direct factor of the change of phase about structure polymer modified asphalt, in general, shearing time will not change the phase structure of polymer, but shearing time will have a certain impact on the phase structure when the phase transition around the critical point.

\section{Acknowledgment}

The authors would like to acknowledge the financial support from the National Natural Science Foundation Committee of China (No.51108402), Scientific Research and Innovation Plans for Postgraduate from Regular Institutions of Higher Learning (CXZZ13_08). We also thank the Engineering Testing Center of Yangzhou University for their support in the experiment.

\section{References}

(1) Shingo K, Shigeru T, Zhang XM, Dong DW, Inagaki N. Compatibilizer role of styrene-butadiene-styrene tri-block copolymer in asphalt. Polym J 2001;33(3):209-13.

(2) Sengoz B, Isikyakar G. Evaluation of the properties and microstructure of SBSand EVA polymer modified bitumen. Constr Build Mater 2007;22: 1897-905.

(3) Polacco G, Stastna J, Biondi L. Relation between polymer architecture and nonlinear viscoelastic behavior of modified asphalt. Eur Polym J 2006;42: 446-57.

(4) Shingo K, Shigeru T, Zhang XM, Dong DW, Inagaki $\mathrm{N}$. Compatibilizer role of styrene-butadiene-styrene tri-block copolymer in asphalt. Polym J 2001;33(3):209-13.

(5) Shingo K, Shigeru T, Zhang XM, Dong DW, Inagaki N. Compatibilizer role of styrene-butadiene-styrene tri-block copolymer in asphalt. Polym J 2001;33(3):209-13.

(6) Sengoz B, Isikyakar G. Evaluation of the properties and microstructure of SBSand EVA polymer Modified bitumen. Constr Build Mater 2007;22: 1897-905.

(7) Polacco G, Stastna J, Biondi L. Relation between polymer architecture and nonlinear viscoelastic behavior of modified asphalt. Eur Polym J 2006;42: 446-57.

(8) Ouyang CF, Wang SF, Zhang Y, Zhang YX. Preparation and properties of styrene - butadiene styrene copolymer/kaolinite clay compound and asphalt modified with the compound. Polym Degrad Stab 2005;87:309 - 17.

(9) Huang Weidong, Sun Lijun. The phase structure of polymer modified asphalt [J]. Highway traffic science and technology, 2001 (10): 1-3.

(10) Nahas. N. C. , BardeL J., Eckmann,B. , ct al, polymer modified asphalts for hiigh performance hot mix pavement binders, AAPT, 1990, 509-525.

(11) Huang Weidong. Polymer modified asphalt, storage stability: the study of microstructure, rheological properties and their relationship [D]. Shanghai: Tongji University,2000

(12) Chen JS, Huang CC. Fundamental characterization of SBS-modified asphalt mixed with sulfur. J Appl Polym Sci 2007;103:2817 - 25.

(13) Polacco G, Stastna J, Biondi L. Relation between polymer architecture and nonlinear viscoelastic behavior of modified asphalt. Eur Polym J2006; $42: 446-57$

(14) Kang Aihong, Zhang Wuhong, Sun Lijun. The preparation method of modified asphalt fluorescence Microscopy sample [J]. Journal of Sichuan : Engineering Science edition, 2012,44(2):154-158.

(15) Kang Aihong, Chen Juan, Sun Lijun Properties of SBS modified asphalt and analysis of micro morphology structure[J]. Journal of PLA University of Science and Technology 2012 (12) Vol.13

(16) Sun Daquan, SBS modified asphalt compatibility studies and engineering properties of [D] Shanghai: Tongji University, 2003.

(17) Liu Zuguang, Wang Shifeng, Wang Dizhen. The structure and properties of SBS modified asphalt [J]. synthetic rubber industry, 2004-01-15,27 (1):39 42.

(18) U. Isacsson, X. Lu. Laboratory investigation of polymer modified bitumens. AAPT,1999. 35-63. 
(19) Blanco R..Rodriguez R.Garduno M.G Castano V. M. Morphology and Tensile properties of styrene butadiene copolymer reinforced asphalt, Journal of applied polymer science 1995, 56, 57-64.

(20) Wang Tao, Cai Hongmei. Shearing process on the effect of SBS modified asphalt's properties [J]. synthetic rubber industry, 2009-01-12,28 (6):454 459. 